\title{
PLGA+HA/ $\beta$ TCP scaffolds embedding simvastatin induced mineral deposition by mesenchymal stem cells
}

\section{Mariane B. Sordi, DDS, MSc; Raissa B. Curtarelli, DDS, MSc; Ariadne C. Cabral da}

Cruz, DDS, MSc, PhD; Ricardo S. Magini DDS, MSc, PhD

Research Center on Dental Implants (CEPID), Federal University of Santa Catarina (UFSC), Florianopolis, Brazil

\section{Background}

The search for bone substitutes that might promote osteoconduction, osteoinduction, and osteogenesis, has fomented research on tissue engineering. Studies based on the association of new approaches, including scaffolds, biological inducers, and mesenchymal stem cells, have shown promising results.

\section{Aim}

The aim of this study was to evaluate the deposition of mineralization nodules in the extracellular matrix induced by simvastatin (SIM) embedded in scaffolds of poly(lactic-coglycolic) acid (PLGA) and biphasic ceramic (BC) composed of hydroxyapatite $(\mathrm{HA})$ and $\beta$-tricalcium phosphate ( $\beta T C P)$.

\section{Methods and Materials}

\section{Samples Preparation}

PLGA : BC $\rightarrow 1: 1$ $\mathrm{BF} \rightarrow 70 \% \mathrm{HA}, 30 \% \beta \mathrm{BTCP}$

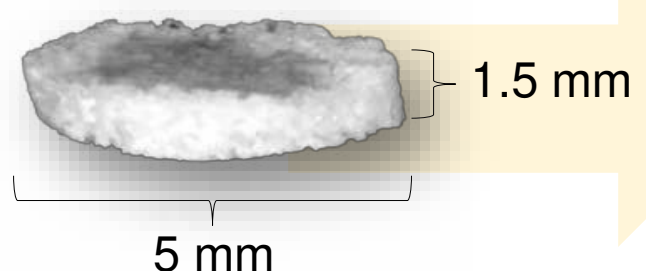

$5 \mathrm{~mm}$

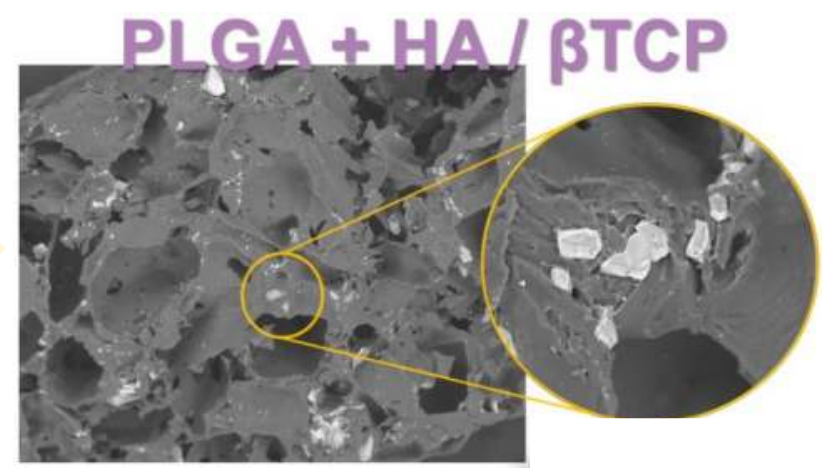

\section{Groups}

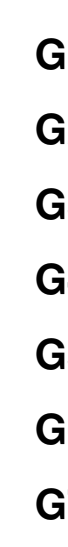

\section{Experimental:}

G1) PLGA+HA/BTCP

G2) $\mathrm{PLGA}+\mathrm{HA} / \beta \mathrm{BCP}+\mathrm{COL}$

G3) PLGA+HA/BTCP+SIM

G4) $\mathrm{PLGA}+\mathrm{HA} / \beta \mathrm{TTCP}+\mathrm{COL}+\mathrm{SIM}$

Control:

G5) SHED in non-osteogenic medium

G6) SHED in osteogenic medium

G7) MC3T3-E1 in osteogenic medium

\section{Mineral deposition assay}

Von Kossa staining methodology was performed after 21 days of cell culture to assess the capacity of scaffolds to induce mineral deposition by SHED. After staining, all groups were taken to optical microscope and photographed, as presented in the Figure 1.

\section{Results}

G6 visibly presented the highest amount of mineralization nodules followed by G3 and G7. G5 presented a high density of eosin-colored cells, but just a few points of mineralization. G1, G2, and G4 showed a variable distribution of both eosincolored cells and mineralization nodules.

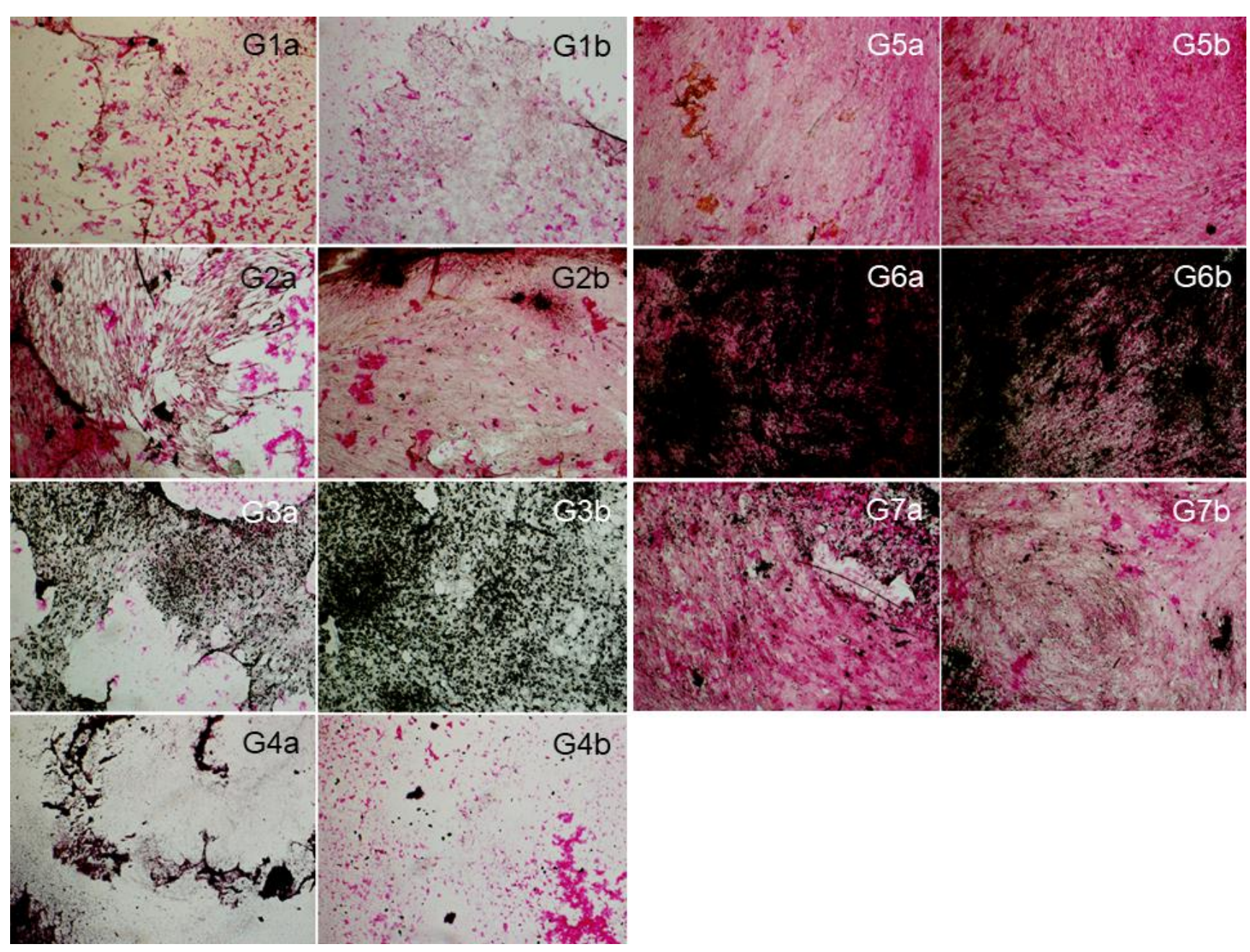

Figure 1. Von Kossa methodology to detect mineralization nodules (colored in brown/black) at day 21 of cell culture.

\section{Conclusions}

$\mathrm{PLGA}+\mathrm{HA} / \mathrm{BTCP}+\mathrm{SIM}$ scaffolds were the most beneficial among experimental groups in regards of deposition of mineralization nodules in the extracellular matrix by SHED. This signalizes SIM embedded in scaffolds of PLGA+HA/BTCP might be a promising biomaterial for tissue engineering, comprehending osteoconduction, osteoinduction, and osteogenesis. Further analyses are suggested to confirm the osteoinductive potential of these scaffolds to be used as bone substitutes for clinical applications.

This study was funded by the International Team for Implantology (ITI) and Brazilian Federal Foundation for Support and Evaluation of Graduate Education (CAPES). 